\title{
ONE CAN HEAR WHETHER A DRUM HAS FINITE AREA
}

\author{
COLIN CLARK ${ }^{1}$ AND DENTON HEWGILL
}

In [1] Clark has obtained an asymptotic formula involving the eigenvalues of the Laplacian operator $-\Delta$ (with zero boundary conditions) on a "quasi-bounded" region $\Omega$ in $R^{n}$. A region is called quasibounded if it cannot contain an infinite family of nonintersecting open solid $n$-spheres of equal size. The formula (valid under certain additional assumptions) is as follows.

$$
N_{\rho}(\lambda) \sim(\lambda / 4 \pi)^{n / 2}(1 / \Gamma(1+n / 2)) \int_{\Omega} \rho(x) d x,
$$

where $N_{\rho}(\lambda)=\sum_{\lambda_{j} \leqq \lambda} \int_{\Omega} \rho(x)\left(\phi_{j}(x)\right)^{2} d x$, in which $\rho(x)$ is an arbitrary nonnegative function in $L_{1}(\Omega)$, and $\left\{\lambda_{j}\right\},\left\{\phi_{j}\right\}$ are the eigenvalues and eigenfunctions, respectively, of the Laplacian in $\Omega$. (This formula has also been derived, under other conditions than in [1], by Hewgill [2].)

The recent paper by $\mathrm{Kac}[3]$ prompts the question: is there some distinction asymptotically between the eigenvalues for a region of finite volume (e.g. a bounded region) and those of a quasi-bounded region of infinite volume?

THEOREM 1. Let $\Omega$ be a quasi-bounded region in $R^{n}$ for which the formula (1) holds. Assume that $\Omega$ has infinite $n$-dimensional volume. Then the function $N(\lambda)=\sum_{\lambda_{j} \leqq \lambda} 1$ satisfies

$$
\lim _{\lambda \rightarrow+\infty} \lambda^{-n / 2} N(\lambda)=+\infty .
$$

This result answers the question prompted by Kac; for the indicated limit is finite (it equals $\left\{(4 \pi)^{n / 2} \cdot \Gamma(1+n / 2)\right\}^{-1}$ times the volume of $\Omega$ ) if $\Omega$ has finite volume.

For the proof, we can take $\rho(x)$ as the characteristic function of some subset $\Omega_{0} \subset \Omega$, of finite but arbitrarily large volume $V$. Then

$$
N(\lambda) \geqq N_{\rho}(\lambda) \sim(\lambda / 4 \pi)^{n / 2}(1 / \Gamma(1+n / 2)) \cdot V,
$$

Received by the editors July 19, 1966.

1 Research sponsored by the Air Force Office of Scientific Research, Office of Aerospace Research, United States Air Force, under AFOSR Grant Nr. AF-AFOSR 379-66. 
so that for sufficiently large $\lambda$,

$$
\lambda^{-n / 2} N(\lambda)>V\left\{(4 \pi)^{n / 2} \cdot 2 \Gamma(1+n / 2)\right\}^{-1} .
$$

Since $V$ is arbitrary, the proof is complete. An alternate but similar proof can be derived from the monotoneity theorem: $\Omega_{0} \subset \Omega$ implies $\lambda_{j}(\Omega) \leqq \lambda_{j}\left(\Omega_{0}\right)$ for all $j$.

Theorem 1 gives a lower bound for the rate of growth of $N(\lambda)$. Using a result of Hewgill, we can also obtain an upper bound. We describe Hewgill's result [2, Theorem 4.3] for the case of two dimensions. Let $\Omega$ be a quasi-bounded region, bounded by three contours: $\gamma_{1}$, the positive $X$-axis; $\gamma_{2}$, the curve $y=\phi(x), x>0$, with $\phi(x)>0$ and $\phi(x) \rightarrow 0$ as $x \rightarrow+\infty$; and $\gamma_{3}$, a bounded contour joining $\gamma_{1}$ and $\gamma_{2}$. Some mild additional assumptions are made concerning smoothness of the function $\phi(x)$. Then: if $\phi^{k} \in L_{1}(0, \infty)$ for some positive integer $k$, the eigenvalues $\left\{\lambda_{j}\right\}$ of the Laplacian in $\Omega$ satisfy

$$
\sum \lambda_{j}^{-2 k}<\infty .
$$

THEOREM 2. Let $\Omega$ be a quasi-bounded region, satisfying the hypotheses of $\left[2\right.$, Theorem 4.3]. Assume that $\phi^{k} \in L_{1}(0, \infty)$ for some integer $k \geqq 1$. Then $\lambda^{-2 k} N(\lambda)$ is bounded.

Proof. Since the sequence $\left\{\lambda_{j}^{-2 k}\right\}$ is nonincreasing and $\sum \lambda_{j}^{-2 k}<\infty$, we have $\lambda_{j}^{-2 k}=O\left(j^{-1}\right)$. Hence $\lambda_{j} \geqq M \cdot j^{1 / 2 k}$ for some $M>0$, and therefore

$$
N(\lambda)=\sum_{\lambda_{j} \leq \lambda} 1 \leqq \sum_{j \leq\left(M^{-1} \lambda\right)^{2 k}} 1=\left[\left(M^{-1} \lambda\right)^{2 k}\right] ;
$$

this shows that $\lambda^{-2 k} N(\lambda) \leqq$ const, as asserted.

If we define

$$
g(\Omega)=\inf \left\{\nu: \lambda^{-\nu} N(\lambda) \text { is bounded in } \lambda\right\},
$$

our results show that (for $n=2$ ) $1 \leqq g(\Omega) \leqq 2 k$. It would be interesting to improve this estimate.

\section{REFERENCES}

1. Colin Clark, An asymplotic formula for the eigenvalues of the Laplacian operator in an unbounded domain, Bull. Amer. Math. Soc. 72 (1966), 709-713.

2. D. E. Hewgill, On Green's function for the Laplace operator in an unbounded domain, Ph.D. Thesis, University of British Columbia, Vancouver, 1966. 1-23.

3. Mark Kac, Can one hear the shape of a drum? Amer. Math. Monthly 73 (1966),

University of British Columbia 